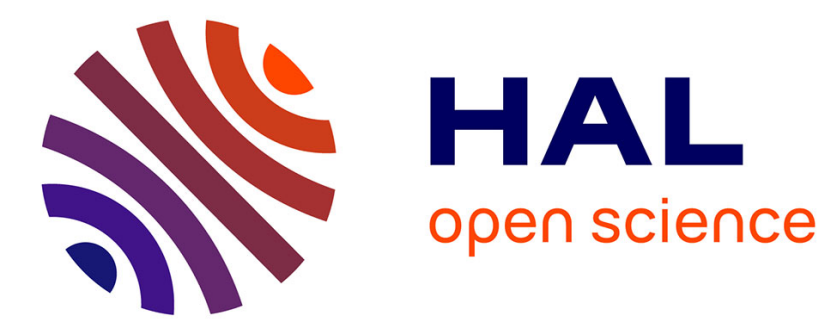

\title{
Software Thread Integration and Synthesis for Real-Time Applications
}

Alexander G. Dean

\section{To cite this version:}

Alexander G. Dean. Software Thread Integration and Synthesis for Real-Time Applications.

DATE'05, Mar 2005, Munich, Germany. pp.68-69. hal-00181496

\section{HAL Id: hal-00181496 \\ https://hal.science/hal-00181496}

Submitted on 24 Oct 2007

HAL is a multi-disciplinary open access archive for the deposit and dissemination of scientific research documents, whether they are published or not. The documents may come from teaching and research institutions in France or abroad, or from public or private research centers.
L'archive ouverte pluridisciplinaire HAL, est destinée au dépôt et à la diffusion de documents scientifiques de niveau recherche, publiés ou non, émanant des établissements d'enseignement et de recherche français ou étrangers, des laboratoires publics ou privés. 


\title{
Software Thread Integration and Synthesis for Real-Time Applications
}

\author{
Alexander G. Dean, Dept. of Electrical and Computer Engineering \\ Center for Embedded Systems Research, North Carolina State University \\ Alex_Dean@ncsu.edu
}

\section{Introduction}

Software Thread Integration (STI) [1] and Asynchronous STI (ASTI) [2] are compiler techniques which interleave functions from separate program threads at the assembly language level, creating implicitly multithreaded functions which provide low-cost concurrency on generic hardware. This extends the reach of software and reduces the need to rely upon dedicated hardware. STI and ASTI are driven by two types of timing requirements: thread-level (e.g. the delay between an event occuring and a service thread running) and instruction-level (e.g. when a specific instruction or code region must begin executing relative to the start of the function or another such instruction or region). These coarse- and fine-grain approach provide a precise method of defining timing requirements. STI provides synchronous thread progress; both functions proceed lock-step. ASTI provides asynchronous (independent) thread progress through the use of lightweight context switches (coroutine calls) between primary and secondary threads. The primary thread has hard-real-time constraints, while the secondary thread is not real-time, or has much longer deadlines.

We assume that instructions take a predictable number of cycles to execute. This implies a straightforward instruction execution pipeline (if used) and a predictable memory system (e.g. the cache is locked, software managed, or not present). These requirements are met for the processors we target: 8 and 16 bit microcontrollers. We target applications with only one hard real-time thread (the primary thread, used for the communication protocol), although recent extensions to STI [3] support multiple hard-real-time primary threads. We have implemented a thread-integrating compiler Thrint which implements many of these analyses and transformations for the AVR architecture, which is 8bit, load/store, and optimized for embedded C code.

\section{STI}

STI targets functions which are independent, data ready and can run to completion. This may require buffering work for one function to ensure it is ready to run when the other is released. For example, in a video controller, graphics ren-

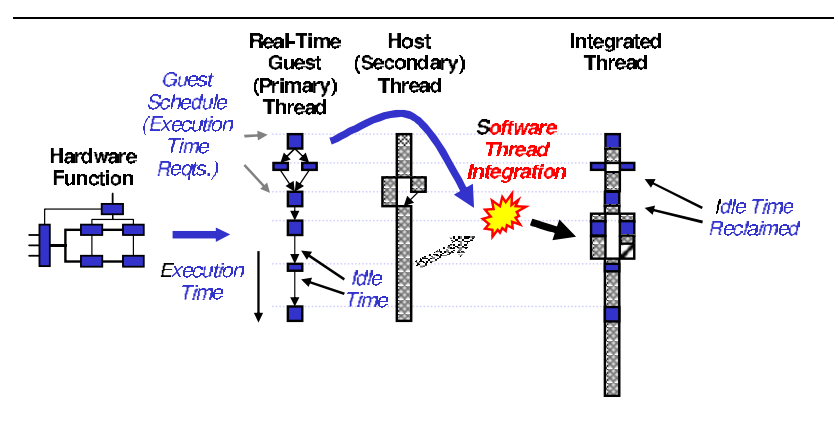

Figure 1. STI interleaves functions at the assembly code level.

dering work is buffered to be available whenever a periodic scan line interrupt service routine runs [3]. STI works by merging two functions into one implicitly multithreaded function, as shown in Figure1. When used for real-time software, it enables the placement of time-critical instructions from one thread so they execute at a specific time relative to the beginning of the integrated function, regardless of the control or data flow characteristics of either thread.

STI begins with building a control-dependence graph representation of the functions to be integrated. We partition the register file between threads at compile time, but more sophisticated register allocation is possible. The bestand worst-case timing of the code is derived statically. This timing is then regularized; execution paths of uneven duration are padded to last the same amount of time (nops and nop loops are used for space efficiency).

Code from one thread can now be moved to execute at given time in the other, using code transformations such as motion, replication, and various for loops (peeling, splitting, guarding, unrolling and fusion). Integration copies code into each path of control flow in a location which ensures it executes at the correct times regardless of the thread progress. Integration involves control-dependence graph traversal (and is hierarchical), and nested transformations are cumulative. Non-looping primary code regions are handled individually. Moving a region into a conditional requires replicating it into both sides, while entering a loop 


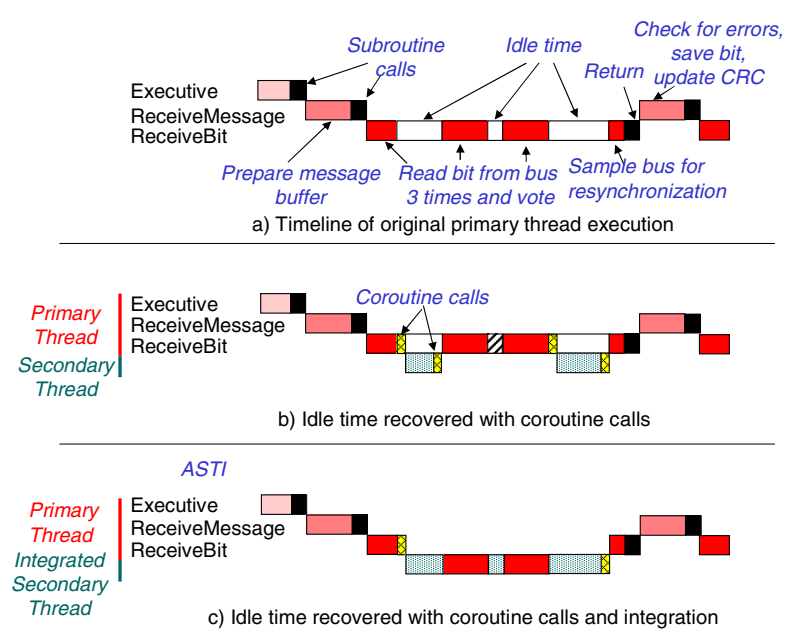

Figure 2. ASTI eliminates most context switches and also recovers finer grain idle time.

requires either guarding the execution with a conditional which triggers on a specific loop iteration or else splitting and peeling the loop. Looping primary function regions are unrolled and treated as non-looping regions unless they overlap with secondary function loops. In that case the overlapping iterations of the two loops are unrolled as needed to match the secondary function loop body work to available idle time in the primary function loop body, increasing efficiency. Integrated code may be also guarded by a conditional test to allow execution based upon mode flags. Next, the timing of the code is analyzed statically again to verify that the transformations placed code properly. In the final stage assembly code is regenerated from the control graph to be assembled and linked with the rest of the application.

\section{ASTI}

ASTI targets applications with frequent context switches and a requirement for asynchronous thread progress. For example, in a software-implemented (bit-banged) communication protocol, the protocol thread must run whenever there is bus activity. However, the idle time in the protocol thread is fragmented into pieces lasting less than a bit or byte. To avoid starvation, other threads are integrated with the protocol thread so they run during the idle time.

ASTI cuts the cost of context switching between the primary (e.g. protocol) and secondary threads by reducing the number of switches, as shown in Figure 2. ASTI uses the idle time $T_{\text {Idle }}$ within a frequently-called primary thread function as a window in which to execute a segment of a secondary thread via a coroutine call (or cocall). There will be $T_{\text {SegmentIdle }}=T_{\text {Idle }}-2 * T_{\text {Cocall }}$ of that time available, given that two cocalls $\left(T_{\text {Cocall }}\right.$ long each) must execute for each segment. After padding to equalize timing of conditionals and loops modulo $T_{\text {SegmentIdle }}$, the entire secondary thread is broken into segments of duration $T_{\text {SegmentIdle. Intervening primary code within the idle time }}$ window is removed and integrated into each segment of the secondary thread, ensuring that running any segment of the secondary thread will still result in the proper intervening primary code executing at the correct times. Coroutine calls are integrated in the secondary thread to ensure it yields control back to the primary thread at the end of the segment, just before the available idle time expires.

\section{Applications}

In the STIGLitz project [3], STI enables an inexpensive $20 \mathrm{MHz}$ AVR 8-bit microcontroller to generate monochrome NTSC video while servicing a high-speed (115.2 kbaud) serial communication link. This system offers graphics rendering speed-ups of $3.99 \mathrm{x}$ to $13.5 \mathrm{x}$ by recovering fine-grain idle time in the display refresh function and using it for video rendering.

We used ASTI to create a bridge between an RS232 link and a software-implemented J1850 automotive embedded network protocol controller [2]. Compared to an interruptbased approach, ASTI results in a secondary thread speedups of 1.56x (message reception) and 1.83x (transmission), enabling the use of an $8 \mathrm{MHz}$ AVR 8-bit microcontroller. An interrupt-based approach would require a $14.7 \mathrm{MHz}$ clock to provide the same performance.

\section{Conclusion}

STI and ASTI extend the performance of commodity low-end microcontrollers through static scheduling techniques, potentially avoiding the need for dedicated hardware or faster clock speeds.

\section{References}

[1] A. G. Dean. Compiling for concurrency: Planning and performing software thread integration. In Proceedings of the 23rd IEEE Real-Time Systems Symposium, Austin, TX, Dec 2002.

[2] N. J. Kumar, S. Shivshankar, and A. G. Dean. Asynchronous software thread integration for effi cient software implementations of embedded communication protocol controllers. In Proceedings of the 2004 ACM SIGPLAN/SIGBED Conference on Languages, Compilers and Tools for Embedded Systems. ACM Press, June 2004.

[3] B. Welch, S. Kanaujia, A. Seetharam, D. Thirumalai, and A. G. Dean. Extending sti for demanding hard-real-time systems. In Proceedings of the International Conference on Compilers, Architectures and Synthesis for Embedded Systems, pages 41-50. ACM Press, November 2003. 